\title{
Crank-Nicolson method for solving uncertain heat equation
}

\author{
Jin Liu ${ }^{1} \cdot$ Yifei Hao ${ }^{2}$ \\ Accepted: 12 November 2021 / Published online: 4 January 2022 \\ (c) The Author(s), under exclusive licence to Springer-Verlag GmbH Germany, part of Springer Nature 2021
}

\begin{abstract}
For usual uncertain heat equations, it is challenging to acquire their analytic solutions. A forward difference Euler method has been used to compute the uncertain heat equations' numerical solutions. Nevertheless, the Euler scheme is instability in some cases. This paper proposes an implicit task to overcome this disadvantage, namely the Crank-Nicolson method, which is unconditional stability. An example shows that the Crank-Nicolson scheme is more stable than the previous scheme (Euler scheme). Moreover, the Crank-Nicolson method is also applied to compute two characteristics of uncertain heat equation's solution-expected value and extreme value. Some examples of uncertain heat equations are designed to show the availability of the Crank-Nicolson method.
\end{abstract}

Keywords Crank-Nicolson method $\cdot$ Liu process $\cdot$ Numerical solution $\cdot$ Heat equation

\section{Introduction}

For dealing with the disturbance or white noise of a dynamic system, in theory, there are two mathematical tools: the differential of Winner process based on probability theory and the differential of Liu process ground on uncertainty theory. The essential difference between these two processes is that the former is a continuous and not Lipschitz continuous, whose increments are normal random variables. By comparison, Liu process is a Lipschitz continuous, whose increments are normal uncertain variables.

If we use the Liu process to model the disturbance in a differential system, then the differential equation evolves into an uncertain differential equation (UDE) (Liu 2008). Nowadays, UDE has been researched by many scholars and has been extensively applied in many areas like finance (Chen and Gao 2013, 2018; Yang and Zhu 2021), dynamic games (Yang and Gao 2016; Zhang et al. 2021) and COVID-19 spread (Lio 2021). In the field of a numerical method for UDE, many ways have been produced, such as the Milne

Yifei Hao

haoyf@ctbu.edu.cn

Jin Liu

liujin229234@163.com

1 College of System Engineering, National University of Defense Technology, Changsha 410073, China

2 School of Business Administration, Chongqing Technology and Business University, Chongqing 400067, China scheme in Gao (2016), Adams-Simpson scheme in Wang et al. (2015), Runge-Kutta scheme in Yang and Shen (2015), Adams scheme in Yang et al. (2015), Euler scheme in Yao and Chen (2013), and Hamming scheme in Zhang et al. (2017). For more other numerical studies, please consult (Kaya et al. 2020; Yokuş 2018; Yokuş and Yavuz 2021). Later, the thought to explain the disturbance with Liu process is spread in the other systems, such as higher-order UDEs in Jia et al. (2018) and Yao (2016), multifactor UDEs in $\mathrm{Li}$ et al. (2015), uncertain fractional differential equations in Zhu (2015), UDE with jumps in Yao (2012), uncertain partial differential equations in Yang and Yao (2017), and uncertain delay differential equations in Barbacioru (2010) and Ge and Zhu (2012).

Uncertain heat equation (UHE) pioneered by Yang and Yao (2017) represents the change of temperature with uncertain disturbance in a given area across time, which is a variety of uncertain partial differential equations. Yang and Yao (2017) supplied the analytic solution for a special type UHE, whose heat source and its diffusion term are functions to time and space but do not depend on its temperature. After that, under the linear growth and Lipschitz conditions, Yang and Ni (2017) showed an existence and uniqueness of the common UHE's solution; Yang (2018) declared that the UHE's solution could be demonstrated by a group of the ordinary heat equation's solutions via $\beta$-path way, and investigated a formula to calculate one characteristic of UHE's solution—expected value; Yang and Ni (2019) stud- 
ied another characteristic of UHE's solution - extreme value; besides, Yang (2019) introduced stability thought of UHE, called stability in measure.

It is needed to design a numerical approach to solve UHE since it is challenging to gain the analytic solution for most UHEs. By $\beta$-path of UHE, Yang (2018) first explored a forward difference Euler method to calculate the solution for a UHE. Nevertheless, this forward difference Euler scheme is conditional stability. We will propose a new numerical scheme named the Crank-Nicolson method to overcome the forward difference Euler scheme's instability limitation. The remainder of this paper is organized as below. Section 2 retrospects some fundamental notions and results in UHE. Section 3 proposes the Crank-Nicolson scheme to compute the UHE's solution. Section 4 designs two algorithms for obtaining two characteristics of the UHE's solutionexpected value and extreme value. Lastly, Sect. 5 gives a brief conclusion.

\section{Preliminaries}

UHE, as a class of uncertain partial differential equations, is investigated by Yang and Yao (2017) to represent the change of temperature with white noise (described by the differential of Liu process) in a region $x \in R$ through time $t \geq 0$. A onedimension UHE defined on a domain $x \times t=R \times[0,+\infty)$ is

$$
\left\{\begin{array}{l}
\frac{\partial H_{x, t}}{\partial t}-a^{2} \frac{\partial^{2} H_{x, t}}{\partial x^{2}}=p(x, t)+q(x, t) \frac{d C_{t}}{d t} \\
H_{x, 0}=\mu(x)
\end{array}\right.
$$

where $H_{x, t}$ represents the temperature at node $(x, t), a^{2}$ is the thermal diffusivity constant, $p(x, t)$ represents the heat source, $q(x, t)$ represents the diffusion term, $C_{t}$ is a Liu process defined on a given uncertainty space, the differential $d C_{t} / d t$ means the white noise with time $t$ described by a Liu process, and $\mu(x)$ is an initial temperature at initial time $t=0$. Yang and Yao (2017) gave that Eq. (1)'s solution is

$$
\begin{aligned}
H_{x, t}= & \int_{-\infty}^{+\infty} D(x-z, t) \mu(z) d z \\
& +\int_{0}^{t} \int_{-\infty}^{+\infty} D(x-z, t-s) p(z, s) d z d s \\
& +\int_{0}^{t} \int_{-\infty}^{+\infty} D(x-z, t-s) q(z, s) d z d C_{s}
\end{aligned}
$$

where

$$
D(x, t)=\frac{1}{2 a \sqrt{\pi t}} \exp \left(-\frac{x^{2}}{4 a^{2} t}\right) .
$$

A general UHE is formulated as

$$
\left\{\begin{array}{l}
\frac{\partial H_{x, t}}{\partial t}-a^{2} \frac{\partial^{2} H_{x, t}}{\partial x^{2}}=p\left(x, t, H_{x, t}\right)+q\left(x, t, H_{x, t}\right) \frac{\mathrm{d} C_{t}}{\mathrm{~d} t} \\
H_{x, 0}=\mu(x) .
\end{array}\right.
$$

If $\mu(x)$ is a bounded function, Yang and $\mathrm{Ni}$ (2017) declared the existence and uniqueness of Eq. (3)'s solution under linear growth and Lipschitz conditions.

Definition 1 (Yang 2018) Let $\beta \in(0,1)$. Equation (3) has a $\beta$-path $H_{x, t}^{\beta}$ if it solves the corresponding ordinary heat equation

$$
\begin{aligned}
& \frac{\partial H_{x, t}^{\beta}}{\partial t}-a^{2} \frac{\partial^{2} H_{x, t}^{\beta}}{\partial x^{2}}=p\left(x, t, H_{x, t}^{\beta}\right) \\
& +\left|q\left(x, t, H_{x, t}^{\beta}\right)\right| \frac{\sqrt{3}}{\pi} \ln \frac{\beta}{1-\beta} .
\end{aligned}
$$

Theorem 1 (Yang 2018) If $H_{x, t}$ and $H_{x, t}^{\beta}$ are the unique solution and $\beta$-path of Eq. (3), respectively, then

$$
\begin{aligned}
& \mathcal{M}\left\{H_{x, t} \leq H_{x, t}^{\beta}, \text { for all } x \text { and } t\right\}=\beta, \\
& \mathcal{M}\left\{H_{x, t}>H_{x, t}^{\beta}, \text { for all } x \text { and } t\right\}=1-\beta .
\end{aligned}
$$

Yang (2018) proved that the $\beta$-path of Eq. (3) is the solution $H_{x, t}$ 's inverse uncertainty distribution, that is,

$\Upsilon_{x, t}^{-1}(\beta)=H_{x, t}^{\beta}$.

For the common UHE (3), it isn't easy to know the analytic solution in most situations. Therefore, Yang (2018) created the forward difference Euler method to obtain $\beta$-path of Eq. (3). Nevertheless, the forward difference Euler method is unstable in some circumstances. This paper will suggest a new numerical scheme, namely the Crank-Nicolson method, which is unconditional stability.

\section{Crank-Nicolson scheme for UHE}

We first review the Crank-Nicolson scheme for ordinary heat equation, and then gives the corresponding Crank-Nicolson scheme for Eq. (3). Considering an initial value problem of an ordinary heat equation

$$
\left\{\begin{array}{l}
\frac{\partial H_{x, t}}{\partial t}-a^{2} \frac{\partial^{2} H_{x, t}}{\partial x^{2}}=p\left(x, t, H_{x, t}\right) \\
H_{x, 0}=\mu(x) .
\end{array}\right.
$$

Let space step be $l$, time step be $\tau, x_{j}=j l(j=$ $0, \pm 1, \pm 2, \cdots)$ and $t_{i}=i \tau(i=1,2, \cdots)$, and $H_{j, i}$ and $p_{j, i}$ be represent the values of $H_{x_{j}, t_{i}}$ and $p\left(x_{j}, t_{i}, H_{x_{j}, t_{i}}\right)$ on 
the node $\left(x_{j}, t_{i}\right)$, respectively. A forward difference Euler scheme is as follows,

$$
\left\{\begin{array}{l}
\frac{H_{j, i+1}-H_{j, i}}{\tau}-a^{2} \frac{H_{j+1, i}+H_{j-1, i}-2 H_{j, i}}{l^{2}}=p_{j, i} \\
H_{j, 0}=\mu\left(x_{j}\right)
\end{array}\right.
$$

that is,

$$
H_{j, i+1}=(1-2 r) H_{j, i}+r H_{j+1, i}+r H_{j-1, i}+\tau p_{j, i}
$$

where $r=\tau a^{2} / l^{2}$. Besides, for the forward difference (6), its truncation error and the stability condition are $O\left(\tau+l^{2}\right)$ and $r \leq 1 / 2$, respectively. The backward difference is as follows,

$$
\left\{\begin{array}{l}
\frac{H_{j, i}-H_{j, i-1}}{\tau}-a^{2} \frac{H_{j+1, i}+H_{j-1, i}-2 H_{j, i}}{l^{2}}=p_{j, i} \\
H_{j, 0}=\mu\left(x_{j}\right)
\end{array}\right.
$$

that is,

$$
(1+2 r) H_{j, i}-r H_{j+1, i}-r H_{j-1, i}-\tau p_{j, i}=H_{j, i-1},
$$

and the truncation error of (8) is $O\left(\tau+l^{2}\right)$, and it is unconditional stability.

We rewrite Eq. (5) as

$$
\left\{\begin{array}{l}
\frac{H_{j, i}-H_{j, i-1}}{\tau}-a^{2} \frac{H_{j+1, i-1}+H_{j-1, i-1}-2 H_{j, i-1}}{l^{2}}=p_{j, i-1} \\
H_{j, 0}=\mu\left(x_{j}\right)
\end{array}\right.
$$

Taking a convex combination with Eqs. (7) and (9) with coefficient $\theta \in[0,1]$ and $1-\theta$, respectively, we have

$$
\left\{\begin{array}{c}
\frac{H_{j, i}-H_{j, i-1}}{\tau}-a^{2}\left[\theta \frac{H_{j+1, i}+H_{j-1, i}-2 H_{j, i}}{l^{2}}\right. \\
\left.+(1-\theta) \frac{H_{j+1, i-1}+H_{j-1, i-1}-2 H_{j, i-1}}{l^{2}}\right] \\
=(1-\theta) p_{j, i-1}+\theta p_{j, i} \\
H_{j, 0}=\mu\left(x_{j}\right),
\end{array}\right.
$$

that is,

$$
\begin{aligned}
(1+ & 2 r \theta) H_{j, i}-r \theta H_{j+1, i}-r \theta H_{j-1, i}-\tau \theta p_{j, i} \\
= & r(1-\theta) H_{j+1, i-1}+[1-2 r(1-\theta)] H_{j, i-1} \\
& +\tau(1-\theta) p_{j, i-1}+r(1-\theta) H_{j-1, i-1},
\end{aligned}
$$

when $\theta \neq 1 / 2$, (11)'s truncation error is $O\left(l^{2}+\tau\right)$, when $\theta=1 / 2$, (11)'s truncation error is $O\left(\tau^{2}+l^{2}\right)$. We note that the recurrence formula (11) is the backward difference when $\theta=1$, and is the forward difference when $\theta=0$.
When $\theta=1 / 2$, the recurrence formula (11) is called CrankNicolson method as follows,

$$
\begin{aligned}
& -r H_{j+1, i}+2(1+r) H_{j, i}-r H_{j-1, i}-\tau p_{j, i} \\
& =2(1-r) H_{j, i-1}+r H_{j+1, i-1}+r H_{j-1, i-1}+\tau p_{j, i-1} .
\end{aligned}
$$

Note that the recurrence formula (11) is an implicit method. For each $i-1$, if we want to get the "next" values of $H_{j, i}$ in time $t_{i}$ for all $x_{j}$, a system of the algebraic equation needs to be resolved. Furthermore, the recurrence formula (11) is unconditional stability when $\theta \in[1 / 2,1]$, and when $\theta \in$ $[0,1 / 2)$, its stability condition is

$2 r \leq \frac{1}{1-2 \theta}$

In other words, Crank-Nicolson method (12) is unconditional stability.

Following, we produce the Crank-Nicolson approach to solve Eq. (3). If $p(\cdot)$ and $q(\cdot)$ meet the linear growth and Lipschitz conditions, and the initial value $\mu(x)$ is bounded, those hypotheses mean that the solution of Eq. (3) is unique. Then, the $\beta$-path of Eq. (3) is a solution of the below equation

$$
\left\{\begin{array}{l}
\frac{\partial H_{x, t}^{\beta}}{\partial t}-a^{2} \frac{\partial^{2} H_{x, t}^{\beta}}{\partial x^{2}}=p\left(x, t, H_{x, t}^{\beta}\right) \\
\quad+\left|q\left(x, t, H_{x, t}^{\beta}\right)\right| \Upsilon^{-1}(\beta) \\
H_{x, 0}^{\beta}=\mu(x)
\end{array}\right.
$$

where

$\Upsilon^{-1}(\beta)=\frac{\sqrt{3}}{\pi} \ln \frac{\beta}{1-\beta}$.

For the sake of convenience, we write

$h\left(x, t, H_{x, t}^{\beta}\right)=p\left(x, t, H_{x, t}^{\beta}\right)+\left|q\left(x, t, H_{x, t}^{\beta}\right)\right| \Upsilon^{-1}(\beta)$.

Then, Eq. (13) can be reduced to

$$
\left\{\begin{array}{l}
\frac{\partial H_{x, t}^{\beta}}{\partial t}-a^{2} \frac{\partial^{2} H_{x, t}^{\beta}}{\partial x^{2}}=h\left(x, t, H_{x, t}^{\beta}\right) \\
H_{x, 0}^{\beta}=\mu(x) .
\end{array}\right.
$$

To obtain the inverse uncertainty distribution or $\beta$-path of UHE's solution, we design the Crank-Nicolson algorithm as below.

Step 0 Domain $O=\{(x, t) \mid 0 \leq t \leq T, 0 \leq x \leq L\}$.

Step 1 Give two positive integers $n$ and $m$, let the space step be $l=L / n$ and the time step be $\tau=T / m$. Write $x_{j}:=j l(j=$ $0,1, \ldots, n), t_{i}:=i \tau(i=0,1, \ldots, m), H_{j i}^{\beta}:=H_{x_{j}, t_{i}}^{\beta}$, and $h_{j, i}^{\beta}:=h\left(x_{j}, t_{i}, H_{j, i}^{\beta}\right)$. 


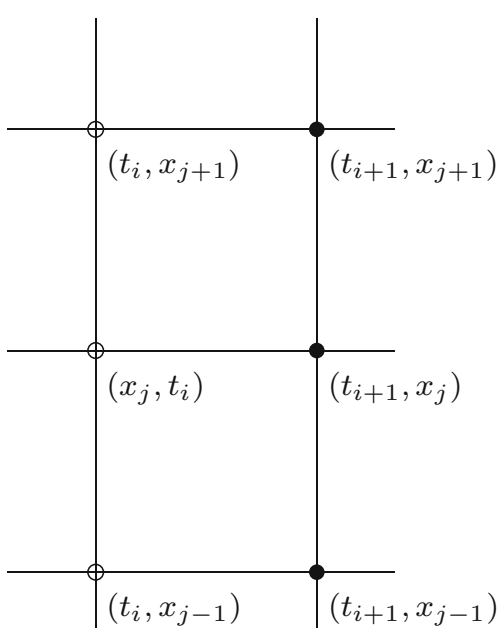

Fig. 1 Node diagram

Step 2 For a $\beta \in(0,1)$.

Step 3 Compute $H_{j, i+1}^{\beta}(j=0,1, \ldots, n, i=0,1, \ldots, m-$ 1) via

$$
\left\{\begin{array}{l}
H_{0, i+1}^{\beta}=(1-r) H_{0, i}^{\beta}+r H_{1, i}^{\beta}+\tau h_{0, i}^{\beta} \\
-r H_{j+1, i+1}^{\beta}+2(1+r) H_{j, i+1}^{\beta}-\tau h_{j, i+1}^{\beta}-r H_{j-1, i+1}^{\beta} \\
\quad=r H_{j+1, i}^{\beta}+2(1-r) H_{j, i}^{\beta}+r H_{j-1, i}^{\beta}+\tau h_{j, i}^{\beta}, \\
\quad j=1,2, \ldots, n-1 \\
H_{n, i+1}^{\beta}=(1-r) H_{n, i}^{\beta}+r H_{n-1, i}^{\beta}+\tau h_{n, i}^{\beta}
\end{array}\right.
$$

where $r=a^{2} \tau / l^{2}, H_{j, 0}^{\beta}=\mu\left(x_{j}\right)$ (Fig. 1 shows the node diagram of the Crank-Nicolson algorithm).

Step 4 For different $\beta$, repeat Steps 2 and $\mathbf{3}$.

Step 5 The $\beta$-path $H_{x, t}^{\beta}$ is obtained.

For each $i$, if we want to get the "next" values of $H_{j, i+1}$ at time $t_{i+1}$ for all $x_{j}$, a system of $n$-1-dimensional algebraic equation must be solved. In others words, the recursion formula in Step 3 is equal to solve an $n$-1-dimensional algebraic equation, and the coefficient matrix of this algebraic equation is tridiagonal.

Example 1 We consider an UHE with $p\left(x, t, H_{x, t}\right)=$ $0, q\left(x, t, H_{x, t}\right)=-e^{-t}$, and $\mu(x)=0$. Reference Yang and Yao (2017) got its analytic solution

$H_{x, t}=-\int_{0}^{t} e^{-s} \mathrm{~d} C_{s}$,

whose inverse uncertainty distribution or $\beta$-path is

$H_{x, t}^{\beta}=\frac{\sqrt{3}}{\pi}\left(1-e^{-t}\right) \ln \frac{\beta}{1-\beta}$.

Firstly, we use the Crank-Nicolson method to get the numerical solution and compare it with the analytical solu-

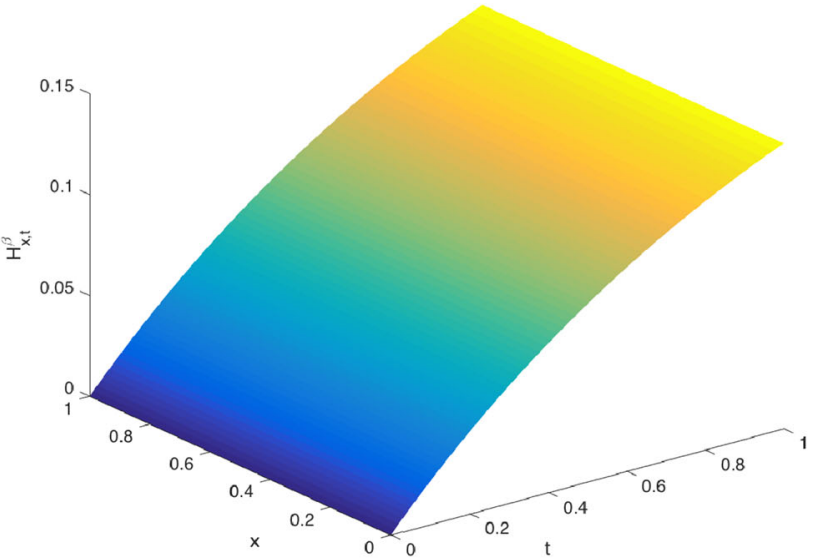

(a) $\beta$-path

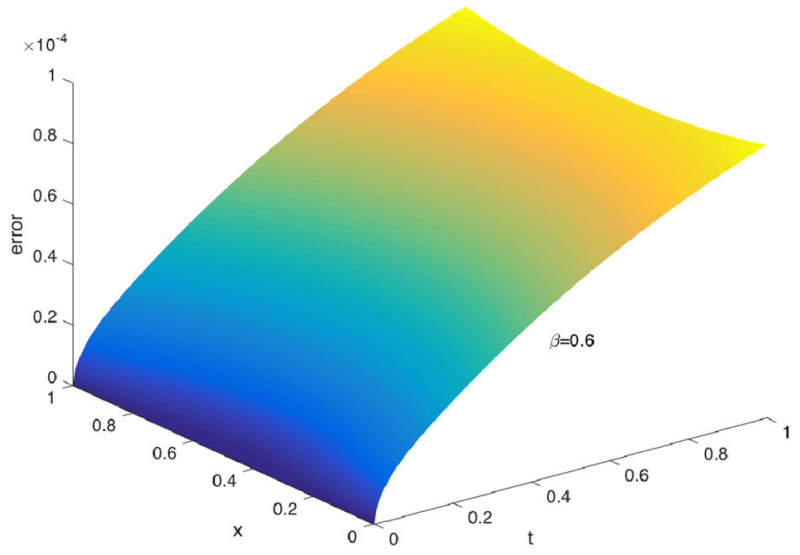

(b) Error surface

Fig. 2 Numerical solution with Crank-Nicolson method

tion. Set $O=\{[0,1] \times[0,1]\}$ and

$\beta=0.6, m=150, n=10$.

Figure 2a shows the $\beta$-path via the Crank-Nicolson method; Fig. $2 \mathrm{~b}$ gives the error surface, which shows this numerical solution is pretty close to the analytical solution. Secondly, summing the absolute difference between this numerical solution and analytical solution on each point $\left(x_{j}, t_{i}\right)$, we get that the Crank-Nicolson scheme's error is 0.0836 , the Euler scheme's error is $8.9007 * 10^{+12}$. Besides, Table 1 shows the results according to the different parameters, we can notice that the Euler scheme is instability in some cases, and the Crank-Nicolson method is stable in the given cases. The reason is that the forward difference's stability condition is $r \leq 1 / 2$, and the Crank-Nicolson scheme is unconditional stability.

Example 2 We consider an UHE with $p\left(x, t, H_{x, t}\right)=$ $e^{-t} \sin x, q\left(x, t, H_{x, t}\right)=1+\cos x$, and $\mu(x)=\sin x$. Let 
Table 1 Error analysis in Example 1

\begin{tabular}{llllllll}
\hline$L$ & $T$ & $\beta$ & $m$ & $n$ & $r$ & $\begin{array}{l}\text { Error } \\
\text { Forward difference }\end{array}$ & Crank-Nicolson \\
\hline 1 & 1 & 0.1 & 800 & 10 & $1 / 8$ & 2.4542 & 0.4470 \\
1 & 1 & 0.2 & 600 & 10 & $1 / 6$ & 1.5491 & 0.2823 \\
1 & 1 & 0.3 & 400 & 10 & $1 / 4$ & 0.9476 & 0.1729 \\
1 & 1 & 0.4 & 200 & 10 & $1 / 2$ & 0.4546 & 0.0833 \\
1 & 1 & 0.6 & 150 & 10 & $2 / 3$ & $8.9007 * 10^{+12}$ & 0.0836 \\
1 & 1 & 0.7 & 125 & 10 & $4 / 5$ & $2.0497 * 10^{+22}$ & 0.1753 \\
1 & 1 & 0.8 & 100 & 10 & 1 & $1.7211 * 10^{+27}$ & 0.2882 \\
1 & 1 & 0.9 & 80 & 10 & $5 / 4$ & $3.4490 * 10^{+28}$ & 0.4595 \\
\hline
\end{tabular}

$O=\{(x, t) \mid 0 \leq t \leq 1,0 \leq x \leq 10\}$, and

$m=200, n=200$.

By Crank-Nicolson method, we have the $\beta$-path or inverse uncertainty distribution of numerical solution. Figure 3 shows the 0.7-path and 0.3-path of numerical solution in Example 2.

Example 3 We consider an UHE with $p\left(x, t, H_{x, t}\right)=$ $2 \sin (3 x), q\left(x, t, H_{x, t}\right)=e^{-t} \sin x$, and $\mu(x)=e^{x} \sin x$. Let $O=\{(x, t) \mid 0 \leq x \leq 1,0 \leq t \leq 1\}$, and

$m=200, n=10$.

By Crank-Nicolson method, we obtain the $\beta$-path of this numerical solution. Figure 4 shows the 0.7-path and 0.3-path of numerical solution in Example 3.

\section{Expected value and extreme value}

In this section, we will use the Crank-Nicolson approach to obtain two characteristics of solution in Eq. (3)-the expected value and the extreme value, and designs the corresponding two algorithms.

\subsection{Crank-Nicolson method for expected value}

Let the function $J(\cdot)$ be monotone and $H_{x, t}$ be a solution of (3). This subsection provides an approach to calculate E $\left[J\left(H_{x, T}\right)\right]$ on a terminal time $T$.

Step $O$ Give an iteration number $M$, a domain $O=\{0 \leq x \leq$ $L, 0 \leq t \leq T\}$, two positive integers $n$ and $m$. Set $\tau=T / m$, $l=L / n, \beta=0$, and $k=0$.

Step 1 Set $k \leftarrow k+1$ and $\beta=k / M$.

Step 2 Compute $H_{j, i+1}^{\beta}(i=0,1, \ldots, m-1, j=$ $0,1, \ldots, n)$ by

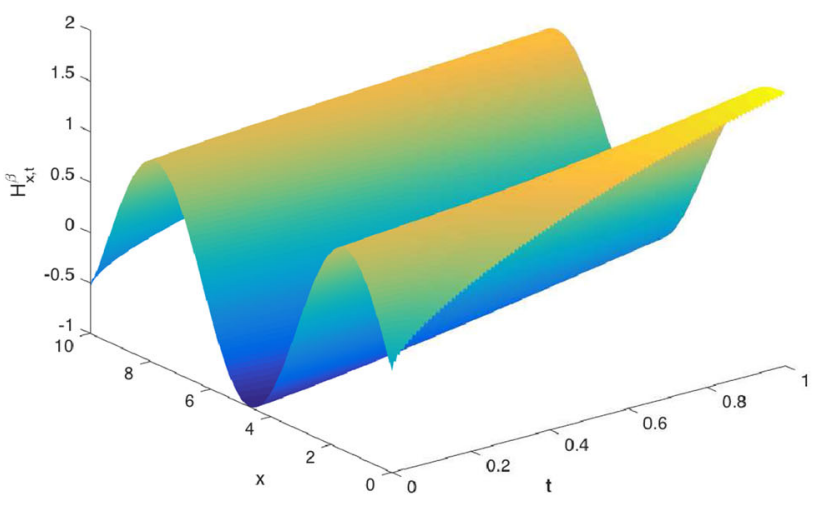

(a) $\beta=0.7$

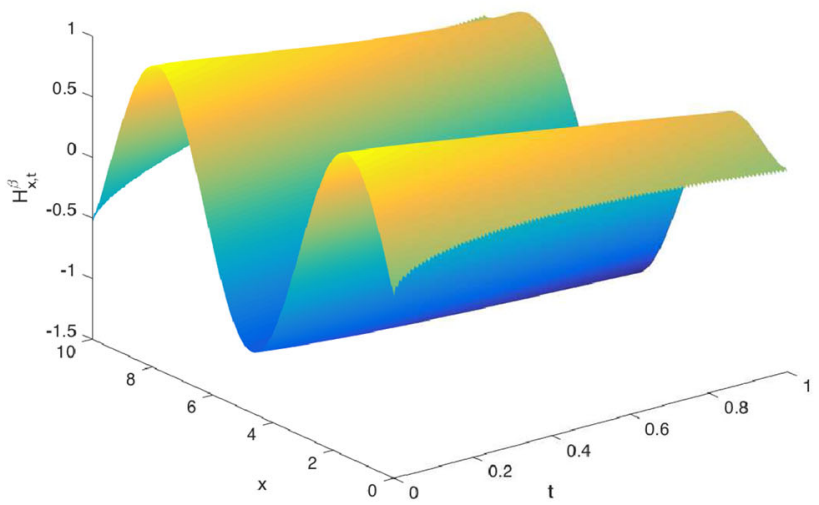

(b) $\beta=0.3$

Fig. $3 \beta$-path in Example 2

$$
\left\{\begin{array}{l}
H_{0, i+1}^{\beta}=(1-r) H_{0, i}^{\beta}+r H_{1, i}^{\beta}+\tau h_{0, i}^{\beta} \\
-r H_{j+1, i+1}^{\beta}+2(1+r) H_{j, i+1}^{\beta}-\tau h_{j, i+1}^{\beta}-r H_{j-1, i+1}^{\beta} \\
\quad=r H_{j+1, i}^{\beta}+2(1-r) H_{j, i}^{\beta}+r H_{j-1, i}^{\beta}+\tau h_{j, i}^{\beta}, \\
\quad j=1,2, \ldots, n-1 \\
H_{n, i+1}^{\beta}=r H_{n-1, i}^{\beta}+(1-r) H_{n, i}^{\beta}+\tau h_{n, i}^{\beta}
\end{array}\right.
$$




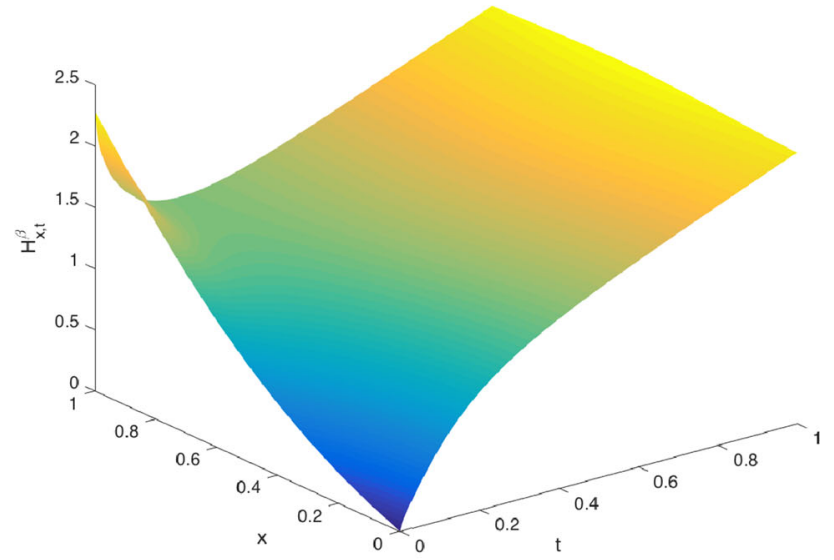

(a) $\beta=0.7$

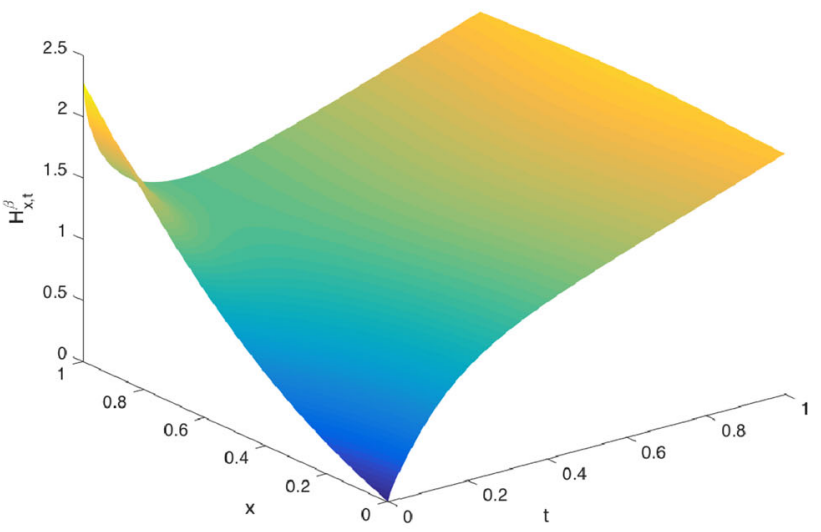

(b) $\beta=0.3$

Fig. $4 \quad \beta$-path in Example 3

where $r=a^{2} \tau / l^{2}, H_{j, 0}^{\beta}=\mu\left(x_{j}\right)$.

Step 3 Obtain $H_{x_{j}, T}^{\beta}(j=0,1, \ldots, n)$. When $k<M$, then go back to Step 1 .

Step 4 Get

$\mathrm{E}\left[J\left(H_{x_{j}, T}\right)\right]=\frac{1}{M-1} \sum_{k=1}^{M-1} J\left(H_{x_{j}, T}^{k / M}\right)$

at time $T$ and point $x_{j}(j=0,1, \ldots, n)$.

Example 4 We reconsider the UHE in Example 1 with $O=$ $\{x \in[0,20], t \in[0,1]\}$, and compute $\mathrm{E}\left[H_{x, t}\right]$. Let the parameters be

$m=200, n=200$.

By the algorithm in Subsection 4.1, we have the expected value $\mathrm{E}\left[H_{x, 1}\right]$, whose curve is shown in Fig. 5 .

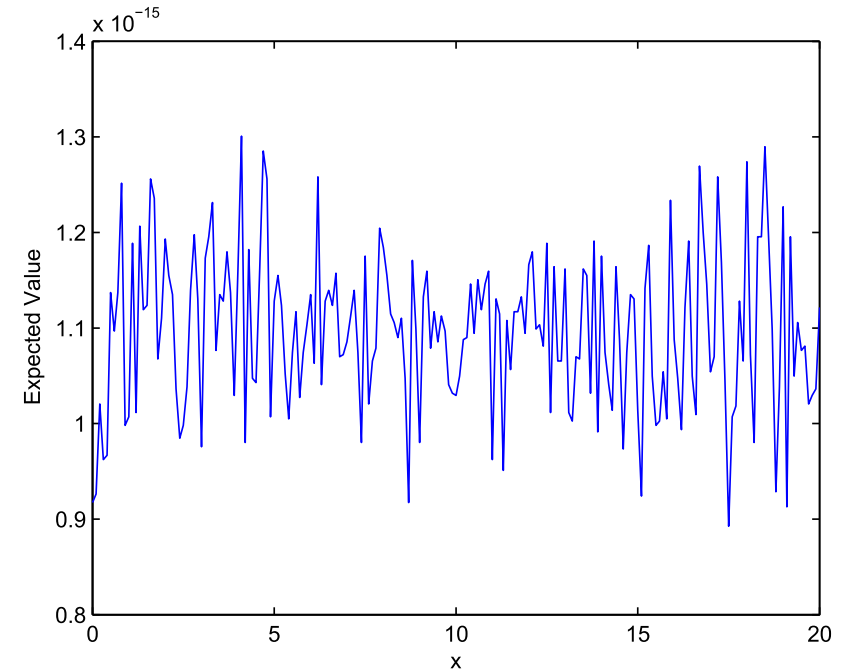

Fig. 5 Expected value $\mathrm{E}\left[H_{x, 1}\right]$ in Example 4

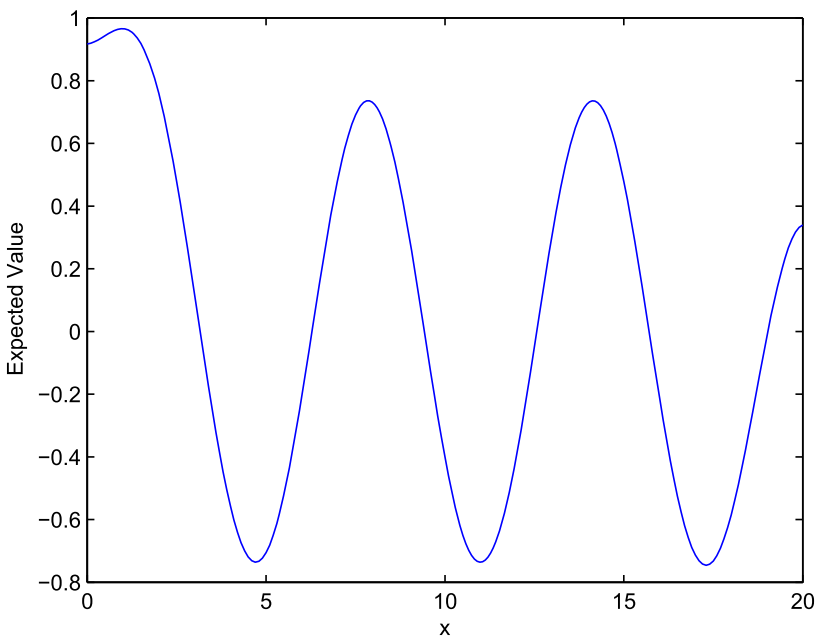

Fig. 6 Expected value $\mathrm{E}\left[H_{x, 1}\right]$ in Example 5

Example 5 We reconsider the UHE in Example 2 with $O=$ $\{x \in[0,20], t \in[0,1]\}$, and compute $\mathrm{E}\left[H_{x, t}\right]$. Let the parameters be

$m=200, n=200$.

By the algorithm in Subsection 4.1, we have the expected value $\mathrm{E}\left[H_{x, 1}\right]$, whose curve is presented in Fig. 6.

Example 6 We reconsider the UHE on Example 3 with $O=$ $\{x \in[0,2], t \in[0,2]\}$, and compute $\mathrm{E}\left[H_{x, t}\right]$. Let the parameters be

$m=400, n=20$.

By the algorithm in Subsection 4.1, we have the expected value $\mathrm{E}\left[H_{x, 2}\right]$, whose curve is shown in Fig. 7 . 


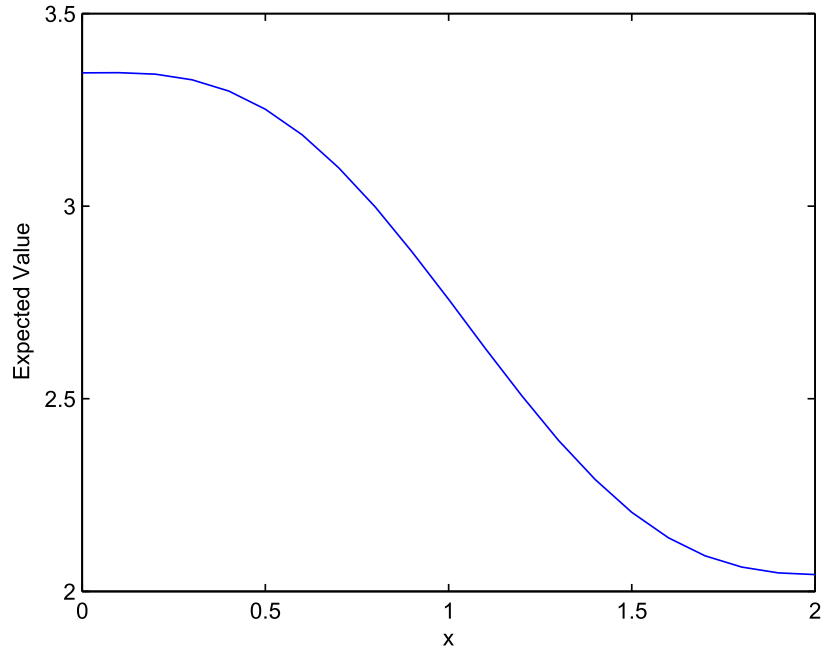

Fig. 7 Expected value $\mathrm{E}\left[H_{x, 2}\right]$ in Example 6

\subsection{Crank-Nicolson method for extreme value}

Let $J_{1}(\cdot)$ or $J_{2}(\cdot)$ be a strictly increasing or decreasing function and $H_{x, t}$ be a UHE's solution under a domain $O=\{x \in[0, L], t \in[0, T]\}$. This subsection proposes the method to obtain the following four extreme values' inverse uncertainty distributions

$$
\begin{aligned}
F_{1} & =\sup _{(x, t) \in O} J_{1}\left(H_{x, t}\right), \\
G_{1} & =\inf _{(x, t) \in O} J_{1}\left(H_{x, t}\right), \\
F_{2} & =\sup _{(x, t) \in O} J_{2}\left(H_{x, t}\right),
\end{aligned}
$$

and

$$
G_{2}=\inf _{(x, t) \in O} J_{2}\left(H_{x, t}\right) .
$$

Step $l$ Give two positive integers $n$ and $m$, let $l=L / n$ and $\tau=T / m$.

Step 2 For a $0<\beta<1$. Set $i=0$,

$$
\begin{aligned}
F_{1} & =\max \left\{J_{1}\left(\mu\left(x_{j}\right)\right), j=0,1, \ldots, n\right\}, \\
G_{1} & =\min \left\{J_{1}\left(\mu\left(x_{j}\right)\right), j=0,1, \ldots, n\right\}, \\
F_{2} & =\max \left\{J_{2}\left(\mu\left(x_{j}\right)\right), j=0,1, \ldots, n\right\},
\end{aligned}
$$

and

$$
G_{2}=\min \left\{J_{2}\left(\mu\left(x_{j}\right)\right), j=0,1, \cdots, n\right\} .
$$

Step 3 Compute $H_{j, i+1}^{\beta}(=0,1, \ldots, n, i=0,1, \cdots, m-$ $1, j$ ) via

$$
\left\{\begin{array}{l}
H_{0, i+1}^{\beta}=(1-r) H_{0, i}^{\beta}+r H_{1, i}^{\beta}+\tau h_{0, i}^{\beta} \\
-r H_{j+1, i+1}^{\beta}+2(1+r) H_{j, i+1}^{\beta}-\tau h_{j, i+1}^{\beta}-r H_{j-1, i+1}^{\beta} \\
\quad=r H_{j+1, i}^{\beta}+2(1-r) H_{j, i}^{\beta}+r H_{j-1, i}^{\beta}+\tau h_{j, i}^{\beta}, \\
\quad j=1,2, \ldots, n-1 \\
H_{n, i+1}^{\beta}=r H_{n-1, i}^{\beta}+(1-r) H_{n, i}^{\beta}+\tau h_{n, i}^{\beta}
\end{array}\right.
$$

where $r=a^{2} \tau / l^{2}, H_{j, 0}^{\beta}=\mu\left(x_{j}\right)$.

Step 4 Set

$$
\begin{aligned}
F_{1} & \leftarrow \max \left\{F_{1}, J_{1}\left(H_{j, i+1}^{\beta}\right), j=0,1, \ldots, n\right\} \\
G_{1} & \leftarrow \min \left\{G_{1}, J_{1}\left(H_{j, i+1}^{\beta}\right), j=0,1, \ldots, n\right\} \\
F_{2} & \leftarrow \max \left\{F_{2}, J_{2}\left(H_{j, i+1}^{1-\beta}\right), j=0,1, \ldots, n\right\} \\
G_{2} & \leftarrow \min \left\{G_{2}, J_{2}\left(H_{j, i+1}^{1-\beta}\right), j=0,1, \ldots, n\right\}
\end{aligned}
$$

and $i \leftarrow i+1$.

Step 5 Repeat Steps 3-5 on $n$ times.

Step 6 The supremum and infimum

$$
\begin{array}{ll}
\sup _{(x, t) \in O} J_{1}\left(H_{x, t}\right), & \inf _{(x, t) \in O} J_{1}\left(H_{x, t}\right), \\
\sup _{(x, t) \in O} J_{2}\left(H_{x, t}\right), & \inf _{(x, t) \in O} J_{2}\left(H_{x, t}\right)
\end{array}
$$

are determined by

$F_{1}, G_{1}, F_{2}, G_{2}$,

respectively.

Example 7 We reconsider the UHE in Example 1 with $O=$ $\{x \in[0,20], t \in[0,10]\}$ and compute the supremum

$$
\sup _{(x, t) \in O} H_{x, t}
$$

and infimum

$$
\sup _{(x, t) \in O} H_{x, t} \text {. }
$$

Let the parameters be

$m=1000, n=200$.

By the algorithm in Subsection 4.2, we have the supremum and infimum of solution, whose curves are shown in Fig. 8. 


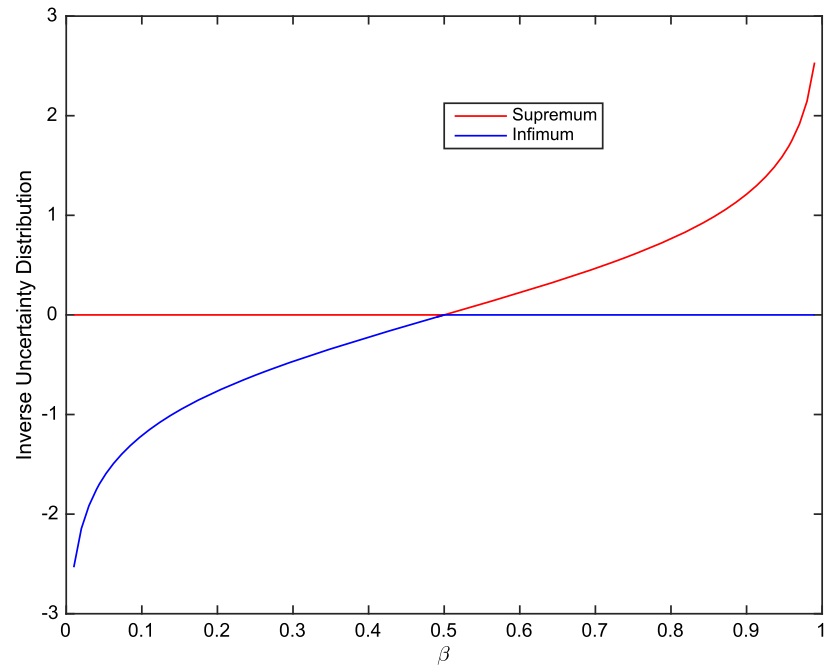

Fig. 8 Extreme values' inverse uncertainty distributions in Example 7

Example 8 We reconsider the UHE in Example 2 with $O=$ $\{x \in[0,20], t \in[0,1]\}$, and compute the supremum

$$
\sup _{(x, t) \in O} H_{x, t}
$$$$
(x, t) \in O
$$

and infimum

$$
\sup _{(x, t) \in O} H_{x, t}
$$

Let the parameters be

$m=200, n=20$.

By the algorithm in Subsection 4.2, we have the inverse uncertainty distributions of supremum and infimum of solution, whose curves are presented in Fig. 9.

Example 9 We reconsider the UHE on Example 3 with $O=$ $\{x \in[0,2], t \in[0,1]\}$, and compute the supremum

$$
\sup _{(x, t) \in O} H_{x, t}
$$

and infimum

$$
\sup _{(x, t) \in O} H_{x, t}
$$

Let the parameters be

$m=400, n=20$.

By the algorithm in Subsection 4.2, we have the inverse uncertainty distributions of supremum and infimum of solution, whose curves are shown in Fig. 10.

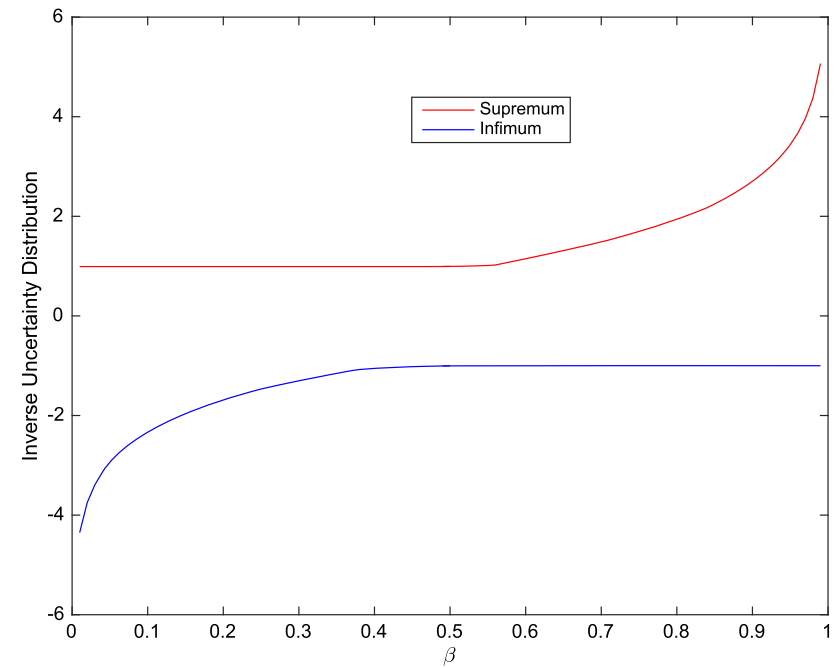

Fig. 9 Extreme values' inverse uncertainty distributions in Example 8

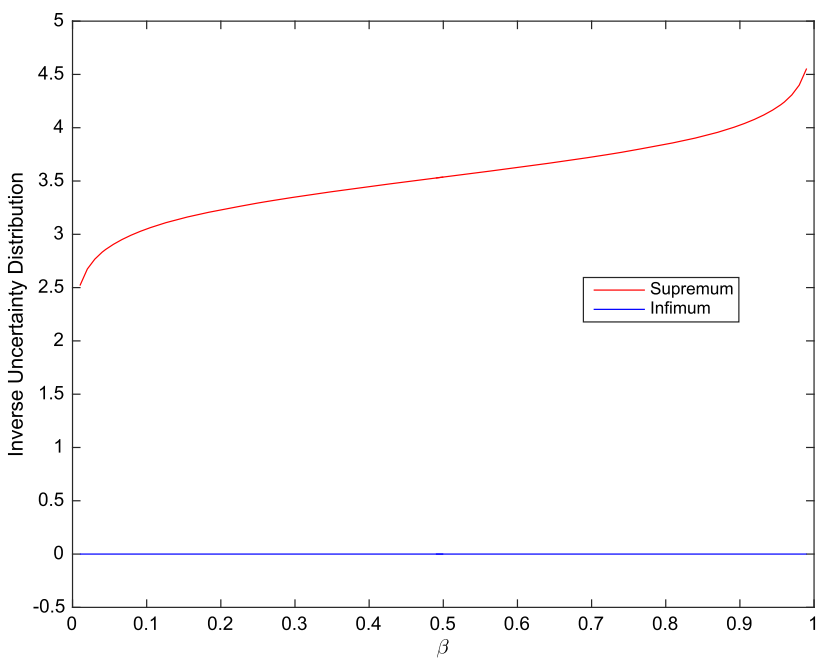

Fig. 10 Extreme values' inverse uncertainty distributions in Example 9

\section{Conclusion}

This paper explored a new approach, namely the CrankNicolson method, to compute the numerical solution of UHE. Compared to this method and the forward difference Euler method proposed in Yang (2018), this method's advantage is unconditional stability. In contrast, the forward difference Euler scheme is conditional stability under $r \leq 1 / 2$. To explain this advantage, we applied the Crank-Nicolson method and the Euler method to Example 1, in which UHE has an analytical solution. Figure 1 shows the $\beta$-path and error surface via the Crank-Nicolson method. Table 1 shows that the Euler scheme is instability in some cases, and the Crank-Nicolson scheme is stable in the given situations. Examples 2 and 3 gave the other UHEs, whose $\beta$-paths are shown in Figs. 2 and 3 via the Crank-Nicolson method. 
Besides, the two algorithms are investigated to compute two characteristics of UHE's solution-the expected value and the extreme value. Examples 4-9 were designed to compute the expected value and extreme value of some UHEs, and the corresponding curves are shown in Figs. 5, 6, 7, 8, 9, and 10 via the Crank-Nicolson method.

Author Contributions All authors contributed to the study's conception and design. In addition, JL and YH performed material preparation, model building, and analysis. JL wrote the first draft of the manuscript, and all authors commented on previous versions. Finally, all authors read and approved the final manuscript.

Funding This work was supported by the National Natural Science Foundation of China under Grant 71701205 and the Humanity and Social Science Youth Foundation of Ministry of Education of China under Grant 19YJC630051.

\section{Declarations}

Conflict of interest The authors claim that they have no conflict of interest.

Ethical approval This study does not include any studies with human participants or animals performed by any of the authors.

\section{References}

Barbacioru I (2010) Uncertainty functional differential equations for finance. Surv Math Appl 5:275-284

Chen X, Gao J (2013) Uncertain term structure model of interest rate. Soft Comput 17(4):597-604

Chen X, Gao J (2018) Two-factor term structure model with uncertain volatility risk. Soft Comput 22:5835-5841

Gao R (2016) Milne method for solving uncertain differential equations. Appl Math Comput 274:774-785

Ge X, Zhu Y (2012) Existence and uniqueness theorem for uncertain delay differential equations. J Comput Inform Syst 8(20):83418347

Jia L, Lio W, Yang X (2018) Numerical method for solving uncertain spring vibration equation. Appl Math Comput 337:428-441

Kaya D, Yokuş A, Demiroğlu U (2020) Comparison of exact and numerical solutions for the Sharma-Tasso-Olver equation. In: Machado J, Özdemir N, Baleanu D (eds) Numerical solutions of realistic nonlinear phenomena. Nonlinear Systems and Complexity, 31 Springer: Cham

Lio W (2021) Uncertain statistics and COVID-19 spread in China. J Uncertain Syst 14:2150008. https://doi.org/10.1142/ S1752890921500082
Li S, Peng J, Zhang B (2015) Multifactor uncertain differential equation. J Uncertain Anal Appl 3 (Article:7)

Liu B (2008) Fuzzy process, hybrid process and uncertain process. J Uncertain Syst 2(1):3-16

Wang X, Ning YF, Tauqir A, Moughal Chen XM (2015) AdamsSimpson method for solving uncertain differential equation. Appl Math Comput 271:209-219

Yang X (2018) Solving uncertain heat equation via numerical method. Appl Math Comput 329:92-104

Yang X (2019) Stability in measure for uncertain heat equations. Discr Contin Dyn Syst Ser B 24(12):6533-6540

Yang X, Gao J (2016) Linear-quadratic uncertain differential game with application to resource extraction problem. IEEE Trans Fuzzy Syst 24(4):819-826

Yang X, Ni Y (2017) Existence and uniqueness theorem for uncertain heat equation. J Amb Intell Human Comput 8(5):717-725

Yang X, Ni Y (2019) Extreme values problem of uncertain heat equation. J Ind Manag Optim 15(4):1995-2008

Yang X, Yao K (2017) Uncertain partial differential equation with application to heat conduction. Fuzzy Optim Decis Mak 16(3):379-403

Yang G, Zhu Y (2021) Critical value-based power options pricing problems in uncertain financial markets. J Uncertain Syst 14:2150002. https://doi.org/10.1142/S1752890921500021

Yang X, Dan A, Ralescu (2015) Adams method for solving uncertain differential equations. Appl Math Comput 270:993-1003

Yang X, Shen YY (2015) Runge-Kutta method for solving uncertain differential equations. J Uncertain Anal Appl 3(Article:17)

Yao K (2012) Uncertain calculus with renewal process. Fuzzy Optim Decis Mak 11(3):285-297

Yao K (2016) Uncertain differential equations. Springer-Verlag, Berlin

Yao K, Chen X (2013) A numerical method for solving uncertain differential equations. J Intell Fuzzy Syst 25(3):825-832

Yokuş A (2018) Comparison of Caputo and conformable derivatives for time-fractional Korteweg-de Vries equation via the finite difference method. Int J Mod Phys B 32(29):1850365

Yokuş A, Yavuz M (2021) Novel comparison of numerical and analytical methods for fractional Burger-Fisher equation. Discr Contin Dyn Syst S 14(7):2591-2606

Zhang Y, Gao J, Huang Z (2017) Hamming method for solving uncertain differential equations. Appl Math Comput 313:331-341

Zhang Y, Gao J, Li X, Yang X (2021) Two-person cooperative uncertain differential game with transferable payoffs. Fuzzy Optim Decis Mak 20:567-594

Zhu Y (2015) Uncertain fractional differential equations and an interest rate model. Math Methods Appl Sci 38(15):3359-3368

Publisher's Note Springer Nature remains neutral with regard to jurisdictional claims in published maps and institutional affiliations. 